LBL- -23040

DE88 000680

HEAVY ION FUSION SYSTEM ASSESSMENT:

FINAL_FOCUS AND TRANSPORT MODEL *

V.O. Brady, A. Faltens, D. Keefe, and E.P. Lee Lawrence Berkeley Laboratory University of California Berkeley, CA $94 \% 20$

J. Hovingh

Lawrence Livermore National Laboratory

Livermore, CA 94550

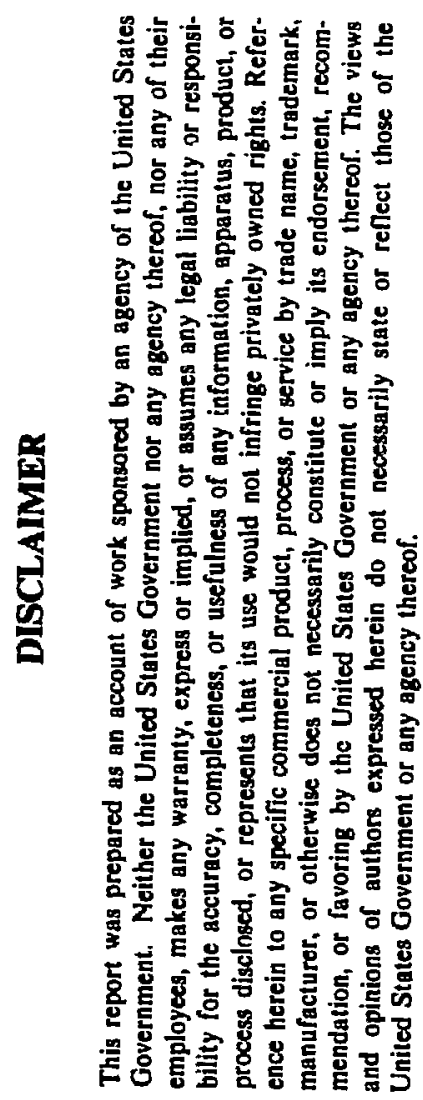

July 1987

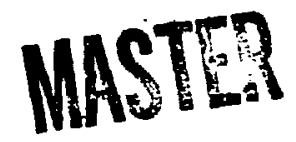

*This was supported by the Office of Energy Research, Office of Prugram Analysis, U.S. Department of Energy, under Contract No. DE-ACD3-765FOUD9B. 


\title{
4.1. HEAVY ION FUSION SYSTEM ASSESSMENT: FINAL FOCUS AND TRANSPORT MODEL*
}

\author{
E. P. Lee, A. Ealtens, and D. Keefe \\ Lawrence Berkeley Laboratory \\ and \\ $\mathrm{J}$. Hovingh
}

Lawrence Livermore National Laboratory

\section{Overview}

At the end of the induction linac the beamlets are laterally separated for transport to final focus and, if necessary, split to provide an increased final number of beamlets on target $\left(\mathrm{N}_{0}\right)$. Splitting may be required for symmetric illumination, to stay within space charge limits, or to provide a sufficient number of beamlets to build up a desired intensity waveform through the use of path length differences. The $N_{0}$ transport channels from the linac to final focus are composed of cold bore superconducting quadrupoles and bends, and possibly higher order magnetic elements which may be needed to control the effects of momentum dispersion and aberrations. The beamlets have a head-to-tail velocity difference on the order of $5 \%$ imposed during the final stages of acceleration, which produces an order of magnitude or more compression during transport, so the final pulse length is in the desired range (5-20 ns) at the pellet. As the beamlets compress, space charge limits in transport are increasingly stressed, with large apertures and close packing of quadrupoles especially pronounced immediately before the final focus magnet set.

The major features of the final focus system are determined largely by the requirements of spot size on the pellet, reactor chamber radius, and the shielding required to reduce the neutron, $x$-ray and gas fluxes from the reactor to an acceptable level. The final focus quadrupole triflets described by $R$. Martin(1) are well suited to serve as the basic final focus components. HIBALL II(2) adopts for this purpose a pair of triplets separated by a pair of weak bends which aid in the removal of line-of-sight neutrons. This general arrangement is also adopted in the present study.

Transport within the reactor vessel has, in most studies, been assumed to take place in near vacuum ( $P<10^{-4}$ Torr $\mathrm{Li}$ ) to avoid disruption by the two-stream instability. (3) Alternatively, a "high pressure window" $(P=1$ - 10 Torr $L i)$ may also be available for stable propagation; (3) its suitability, however, in a reactor/beam line environment in very unclear because of the large gas load that may stream back into the final focus lenses. HIBALL II specifies $P<3 \times 10^{-6}$ Torr $\mathrm{Pb}$ vapor to avoid the stripping of electrons from the beam ions (which might cause spot size blow up through the interaction with the beam's electric field). Unfortunately, several attractive reactor concepts considered in the present study (HYLIFE(4), CASCADE(5), WET WALL(6)) have residual gas pressures in the range $10^{-2}-10^{-3}$ Torr $\mathrm{Li}$ at reasonable repetition rates $(2-10 \mathrm{~Hz})$; this pressure must be taken into account with regard to both transport in the reactor and the leaking of gas into the final focus beam lines. Recent calculations( 7 ) indicate that, contrary to earlier conclusions, the two-stream instability is benign at these pressures due to the detuning effect of beam convergence. 
To produce the small spot radius $\left(r_{s}\right)$ on target, the $f$ inal beamlet unnormalized emittance ( $\varepsilon$ ) must satisfy the condition $\varepsilon<r_{s} \theta$, where $\theta$ is the beamlet convergence cone half-angle. For the typical parameters $r_{\mathrm{S}}=3 \mathrm{~mm}, \theta=10 \mathrm{mr}$, we require $\varepsilon<3 \times 10^{-5} \mathrm{~m}-\mathrm{r}$. This value appears to be achievable using high brightness ion sources( 8 ) and is well matched to the desired range of normalized emittance for a cost-optimized accelerator. Allowance must also be made for the effects on spot size of momentum spread $( \pm \Delta p)$, various forms of beam jitter, and space charge induced blow-up. A Einal focus system composed of a pair of triplets has dispersion at the peilet which leads to an increased spot radius $\Delta r_{s} \approx 8 L \theta(\Delta p / p)$, where $L$ is the distance from the center of the final magnet to the pellet. Unless this is corrected by special beamline elements, it is desirable to limit the momentum spread to the range $\Delta p / p \leq \pm 10^{-3}$. This is a severe requirement on the accelerator system (which must produce a value less than $\sim \pm 10^{-4}$ prior to compression).

In summary, the required small spot size on the pellet is met by low specified emittance, small momentum spread, and a set of other focal and reactor constraints which are only partially understood at present. The convergence cone half-angle, $\theta$, is set at a value which is determined by tradeoffs among economic and physical factors. Factors driving a low value of $\Theta$ are dispersion, aberrations, magnet costs, reactor economics, shielding and beamline vacuum. Factors driving a large value of $\Theta$ are the emittance limit, space charge limits, and jitter control. The typical range of reactor values $(\theta=5-20 \mathrm{mr})$ is the result of compromise among these factors. Aside from the spot size condition, it is desired to make the normalized emittance relatively large $\left(\varepsilon_{n}=B \gamma \varepsilon>10^{-5} \mathrm{~m}-r\right)$ since transportable curcent is found to vary as $\varepsilon_{n}^{2 / 3}$, and too small a value of $\varepsilon_{n}$ could result in an unreasonably large number of beamlets in the high energy portion of the linac ( $N$ increasing above $\sim 24$ ).

\section{Pulse Compression}

At the end of acceleration the ion pulse is typically 100-400 ns in length, which is well matched to the bandwidth of the accelerator pulse forming system. Subsequent reduction to the desired 5-20 ns length desired for the fusion pellet implosion is achieved by the mechanism of drift compression in the transport lines leading to the final focus system. If the initial pulse length (in $\mathrm{m}$ ) is $\ell_{0}$ and the drift lines have length $z_{0}$. then a head to tail velocity tilt of approximately

$$
\frac{\Delta v}{v}=\frac{P_{0}}{Z_{0}}
$$

must be applied in the final stages of acceleration. If, for example, $l_{0}=20 \mathrm{~m}$ and $z_{0}=400 \mathrm{~m}$ then the pulse tail must move $5 \%$ faster than the head in the transport lines. There are several important considerations in this approach:

(a) The bends in the transport system must handle the velocity tilt and space charge with a minimum of dispersive effects. There have been only rudimentary (but encouraging) calculations of a design to accomplish this. 
(b) Longitudinal space charge forces reduce the velocity tilt as the pulse compresses; the initial tilt must be large enough that it is not entirely removed before the desired final pulse length is reached.

(c) Any residual tilt remaining in the pulse at the time of final focus will result in a potentially severe second order chromatic aberration at the pellet. It is assumed that this can be compensated by the use of rapidly pulsed quadrupoles in an upstream location. These pulsed quadrupoles would impose a time dependent envelope oscillation which would cancel the time dependent aberration resulting from the remaining tilt.

(d) The generation of longitudinal momentum spread by the inhomogeneous fields acting during compression is minimal(ideally $\Delta p / p \leq$ $10^{-3}$ in final focus). A recent, and preliminary particle-in-ce $1 \bar{l}$ simulation of compression dynamics indicates that final momentum spread can be on the order of $10 \%$ of the initial tilt( $(9)$. This is larger than desired by a factor of several.

The System Assessment model assumes the favorable resolution of these concerns. However, the role of space charge in removing tilt appears to be sufficiently fundamental that a scale law has been devised for the system Model. We adopt the approximate representation of longitudinal electric field

$$
E_{z}=-\frac{g}{\gamma^{2}} \frac{\partial \lambda}{\partial z} \text {, }
$$

where $\lambda(z, t)$ is the line charge density of the beamlet and

$$
g=\frac{1}{2}+\log _{e}\left(\frac{b^{2}}{a^{2}}\right) \approx 1.3
$$

is a geometric factor involving the ratio of channel radius $b$ and beam radius $a$. Then a pulse with parabolic longitudinal profile must have initial tilt

$$
\left(\frac{\Delta v}{v}\right)_{0}^{2} \geq 12 \text { g K }\left(1-\frac{\ell_{f}}{l_{0}}\right) \text {. }
$$

Here $\ell_{0}$ and $\ell_{f}$ are initial and $f$ inal pulse lengths and $K$ is beamlet perveance in final focus

$$
K=\frac{2 \text { ge I }}{(B r)^{3} M c^{3}}\left(4 \pi \varepsilon_{0}\right)^{-1}
$$


The system code generally uses the limiting value $K=\theta^{2}$ (see discussion of perveance limit given below) where $\theta$ is the convergence half angle in final focus. Inserting the typical values $\theta=.01 \mathrm{rad}$ and $\ell_{E} / \ell_{0}=.05$ we find the tilt condition

$$
\left(\frac{\Delta v}{v}\right)_{0}>\left[(12)(1.3)\left(10^{-4}\right)(.95)\right]^{1 / 2}=.038
$$

This is an appréciable, but probably not unacceptable, value. A residual momentum spread of $\pm 5 \%$ of this tilt would be double the value of \pm .001 assumed in the model. Thus we are somewhat optimistic on this point.

\section{Perveance Limit}

The minimum number of $f$ inal beamlines $\left(N_{0}\right)$ required to transport the beam ions to the fusion pellet with radius $r_{s}$ can be estimated from a consideration of space charge effects in the reactor chamber. First consider the situation in which the beamlets transverse the chamber in vacuum and that space charge is the dominant defocussing effect. Then the beam envelope equation is

$$
\frac{d^{2} a}{d s^{2}}=\frac{R}{a}
$$

where $K$ is the beamlet perveance:

$$
K=\frac{2 \text { Iqe }}{(B Y)^{3} m_{0} c^{3} A 4 \pi \varepsilon_{0}}=\frac{21 g}{\left(B_{Y}\right)^{3} A\left(31 \times 10^{6} \mathrm{amp}\right)}
$$

The perveance is a dimensionless measure of beamlet current. The minimum beamlet radius resulting from this equation is

$$
r=a_{1 e n s} \exp \left(-\theta^{2} / 2 K\right)
$$

where $\theta$ is the convergence cone half angle and

$$
a_{\text {lens }}=\text { L } \theta
$$

is the beam radius at the final lens. For a power reactor, we expect $\mathrm{L}=5-10 \mathrm{~m}, \theta=10-20 \mathrm{mr}$, and $\mathrm{r}=2-4 \mathrm{~mm}$. To make space charge negligible, 
we therefore require, in the absence of neutralization

$$
k \leq(.1) \theta^{2}
$$

This condition leads to unacceptably large numbers of beamlets when the charge state exceeds $q=2-3$, so some degree of neutralization must be invoked in general. The figure adopted in the HIFSA study is $90 \%$ neutralization, either from the ionization of residual gas or co-injection of electrons. Recent calculations by 0150 (10) indicate that the ion pulse is able to trap an electron cloud of sufficient density and low enough temperature to accomplish this. Thus assuming neutralization we adopt the condition

$$
K \leq \theta^{2}
$$

The final number of beamlets $N_{0}$ can be related to the total energy delivered to the pellet (W), beamlet current (I), and pulse-length ( $T_{p}$ ) by

$$
N_{0}=\frac{W}{I_{p} T_{0}^{/ q e}} \simeq \frac{4 W e^{2} q^{2}}{K(B Y)^{5} A^{2} m_{0}^{2} c^{5} 4 \pi \varepsilon_{0} T_{p}} \text {. }
$$

A convenient formula for $\mathrm{N}_{0}$ is

$$
N_{0}=(.138)\left(\frac{q}{A}\right)^{2} \frac{W}{K(B \gamma)^{5} \tau_{n s}} \text {, }
$$

where final pulse length is given in nanoseconds ( $\tau_{n s}$ ) and we used the non-relativistic formula $\mathrm{T}_{0}=\left(\beta_{\gamma}\right)^{2} \mathrm{Mc}^{2} / 2$. For the typical case $\left(q=3, A=200, W_{M J}=4, T_{n s}=10, K=2.25 \quad x \quad 10^{-4}, \quad B r=.33\right)$, we get $\mathrm{N}_{0}=14.1$, which rounds up to $\mathrm{N}_{0}=16$ for symmetric two-sided illumination.

\section{Transport Lines}

Transport lines Erom the Linac to the final focus train serve a variety of purposes:
a. drift compression,
b. bends matched to final focus configuration,
c. delay lines for multiple pulse linac,
d. correction and compensation of aberrations and dispersion,
e. pulse splitting.

It has not been feasible to make detailed estimates of cost, length and configuration which accommodate all of these features. A crude estimate of cost, which is proportional to the length and total number of the transport 
lines is used in the system code:

$$
\cos t / m=(5000 \$)\left[1+\left(\frac{\left[B_{\rho}\right]}{200 \mathrm{~T}-m}\right)^{2}\left(\frac{k}{2 \times 10^{-4}}\right)^{2}\right] \text {. }
$$

The actual perveance, of course, is much lower than the final value (K) during most transport; but. the dependence of radius on current is approximately correct. The quantity $[\mathrm{BP}]$ is the magnetic rigidity of the ions $([B \rho]=B Y M c / q e)$. Tunnel costs are a separate item in. the system code.

Any splitting zones required to convert the $N$ accelerated beamlets into $N_{0}$ final beamlets are costed at $20 \mathrm{k} \$ / \mathrm{m}$ for a total bend of $\pi / 8$ radius:

$$
\frac{\cos t}{\text { split }}=\frac{20,000 \$}{m} \quad\left(\frac{[B e]}{2 \Gamma}\right)\left(\frac{\pi}{8}\right) .
$$

The length of beam lines is highly dependent on both the focal geometry and the final focus train length $(8 \mathrm{~L})$. Where $L$ is the focal length of lenses close to the reactor (see sect. 6). A rough matchup of geometry has motivated the following formulas (length per transport line)

Single sided Illumination:

line length $=18 \mathrm{~L}$

Double sided Illumination:

$$
\text { line length }=9 L+\left(\frac{3 \pi}{2}-1\right) P .
$$

where

$$
\rho=[B \rho] / 2 T
$$

is the average radius of curvature in the bend system. The total length is not allowed to be shorter than $18 \mathrm{~L}$.

Uniform Illumination:

$$
\text { line length }=20 \mathrm{~L}+2 \pi \rho .
$$

This is necessary to carry beanlets to the back side of the reactor. Double Pulse delay lines:

$$
\text { line length }=18 \mathrm{~L}+B c \tau_{d}
$$

where delay time $\tau_{d}=10^{-5} \mathrm{~s}$ is assumed. The second pulse lines have length 18L.

\section{Transportable Current}

Immediately prior to final focus the beamlet current (as computed from the perveance limit) can potentially exceed the Maschke transport 
limit. (11) To insure against this possibility, the final beamlet current (I) is tested against this limit, allowing a factor of two for compression during final focus; that is, $I \leq 2 I_{l i m i t}$ is allowed.

The specific formula for limiting current used here(12) is the general form

$$
\left.I_{\text {limit }}=\left(2.89 \times 10^{6} \mathrm{Amp}\right)\left(1-\frac{\sigma^{2}}{\sigma_{0}^{2}}\right)\left[\sigma_{0}^{4}(B Y)^{5} n^{2}\left(\frac{A}{q}\right)\left(\frac{\sigma_{n}}{\sigma}\right)^{2} B^{2}\right]^{1 / 3}, 17\right)
$$

where $\sigma_{0}$ and $\sigma$ are the normal and depressed tunes of the transport lattice, $\eta$ is the quadrupole occupancy factor and $B$ is the quadrupole field at the beam edge. We adopt the maximum reasonable values for these quantities as understood at present:

$$
\begin{aligned}
& \sigma_{0}=85^{\circ}=1.48 \mathrm{rad}, \\
& \sigma_{0} / \sigma=10, \\
& \eta=.5 \\
& B=3 \mathrm{~T}
\end{aligned}
$$

to obtain

$$
I<2 I_{\text {Iimit }}=\left(45.2 \times 10^{6} \mathrm{Amp}\right)(B Y)^{5 / 3} \cdot\left(\frac{A}{q}\right)^{1 / 3} \varepsilon_{n}^{2 / 3}
$$

For the typical values $A / q=67, B \gamma=.33, \varepsilon_{n}=10^{-5}$ rad-m, we get $I<13.4 \mathrm{kA}$, (which is generally satisfied).

\section{Einal Focus 'Components and Their Arrangement}

The final focus system rescribed in the HIBALL II report(2) serves as a model for HIFSA. The esset،cial layout consists of two quadrupole triplets separated by a pair of weak bends $\left(\sim 3^{\circ}\right.$ each). The first triplet expands the beamlet to a radius of $\sim 20-40 \mathrm{~cm}$ and the second triplet focusses it onto the pellet. The bends allow line-of-sight neutrons to be removed from the beamline. If neutrons were absent then a single quadrupole quadruplet would be adequate. The magnets are assumed to be superconducting with a warm bore (for system efficiency), with the possible exception of the final quadrupole, which may be an iron dominated electromagnet in order to withstand the neutron flux. A rough model of the system optics is obtained by taking the focal length of all six quadrupoles equal to the distance (L) from the middle of the final magnet to the fusion pellet. The beam radius in the final magnet is $L \theta$, and the maximum radius in the train is $3 L \theta$, which is reached in the second and $f$ ifth quadrupoles. Space charge effects are incorporated in this design only in the crude sense that magnet gradients are increased by a factor of $\sqrt{2}$ over the values required in the absence of space charge. Magnet lengths are set equal L/2 except for the 
final quadrupole, which is generally shorter ( L/4) in order to reduce the final focal length. Total length for the train is set at 8L (generally in the range 50-100 $\mathrm{m}$ ), which is $50 \%$ occupied by magnets. The superconducting wire is set back to a radius of twice that of the beam to allow room for shielding, cooling and thermal insulation.

For the described final focus layout the spot radius is given by

$$
r_{s}^{2} \approx \frac{\varepsilon^{2}}{\theta^{2}}+(8 L \theta \Delta P / P)^{2}
$$

assuming aberrations and space charge in final transport can be neglected. The minimum spot radius is obtained with

$$
\theta=\left(\frac{\varepsilon}{8 L \Delta P / P}\right)^{1 / 2}
$$

For the system cost algorithm we use the formula

$$
\frac{M \$}{\text { Train }}=\underset{\text { cost }}{\text { enclosure }}+(1.2)\left(.5 M \$+8 \frac{M \$}{\text { magnet }}\right)
$$

The enclosure cost is $\$ 2830 / \mathrm{m}$ for a cut and cover tunnel. The factor of (1.2) includes all shielding, utilities, power supplies, etc. A .5M\$ price is included to cover the transition zone in which the gas load is reduced, and the fac:or of 8 reflects the number of magnets per iine. The cost per magnet estimate is based on the third quadrupole, which is of medium size compared with the others. Its wire radius is $4 \theta \mathrm{L}$, with field at the wire $B_{C}=8 \sqrt{2}\left[B_{\rho}\right] \theta / L$, where $[B \rho]$ is the magnetic rigiaity of the beam. Generally, values of $B_{c}$ obtained range from $\sim(1.0-5.0) T$, but an upper limit of $8 \mathrm{~T}$ at the wire is imposed.

The cost per magnet is a modification of the formula used in the induction linac design code LIACEP, (described elsehwere in the HIFSA report), which in turn was based on a 1979 survey of existing design estimates. It is

$$
\frac{M \$}{\text { magnet }}=\left(77 \times 10^{-4}\right)\left(.285 \ell_{m}+.43\right)\left(8+76.8 r_{c}+81.1 r_{c} B_{c}\right) \text {, }
$$

where $r_{c}=(4 \theta \mathrm{L}-.04 \mathrm{~m})$. A point check of this formula with a current SSC magnet cost code gave agreement within $10 \%$ for a large bore quadrupole.

\section{Geometric Aberrations}

During final focus significant deviations from paraxial ray optics may appear if the beam is focussed too abtuptly. These deviations appear as a third-order effect in the formulation of beam transport, and their 
consequence is to distort the bean spot shape such that intensity is reduced. D. Neuffer has given the condition(13) for this distortion to be tolerable, assuming the spot radius is determined by emittance $\left(\Sigma_{s}=\varepsilon / \theta\right):$

$$
\varepsilon<(0.15) r_{s}^{5 / 4} p^{-1 / 4} \text {, }
$$

where $\rho$ is radius of curvature of a particle at the beam edge in the final focus quadrupole fields. In the present study, momentum spread and possibly space charge also play a role in determining spot radius, so Neuffer's formula is modified by substituting $\varepsilon=\theta r_{s}$ to obtain the equivalent form

$$
\theta<.15\left(r_{s} / \rho\right)^{1 / 4}
$$

which should apply in the more general situation. This formula is used in the system code to place an upper limit on $\theta$. The typical maximum values are in the range 15-30 mr and may be slightly conservative since some measures such as the insertion of octupoles can be taken to reduce such aberrations.

Aberrations in final focus associated with closeness of the beam envelope to the magnet pole tips are generally not expected to be a problem since in most cases the finai focus coils are moved well back from the aperture (by a factor of two) in order to allow space for shielding and insulation. In addition, the magnets are operateu at a fixed strength, thus permitting flexibility in design that could minimize aberrations due to field errors, fringe fields etc.

\section{Gas Load in Final Focus Lenses}

It is essential that the residual pressure in the reactor chamber $\left(P \sim 10^{-2}-10^{-3}\right.$ Torr $\mathrm{Li}$ ) be attenuated by a large factor between the reactor and the final focus train. Otherwise the bulk of the beam ions are stripped before the focal process is completed and are thereby misdirected. It is assumed in the study that this can be achieved with a combination of fast shutters and pumping in a transition region of about $1.0 \mathrm{~m}$ in length located between the final quadrupole and the reactor shield. Some estimates of the requirements follow.

The stripping length $l_{S}=\left(n_{g} \sigma_{S}\right)^{-1}$ should be at least $300 \mathrm{~m}$ in the final magnet if bear loss is to be kept below $\sim 1 \%$. There is further pumping upstream so $l_{s}$ gets longer rapidly as one moves away from the reactor. The stripping length is approximately (see subsection 9.2)

$$
\ell_{S} \approx \frac{1.0 \mathrm{~cm}}{\text { PTorr }_{\text {Tor }}}\left(\frac{92}{Z_{i}}\right),
$$

we require (for $\mathrm{u}^{238}$ on $\mathrm{Li}$ ) 


$$
P \leq 3 \times 10^{-5} \text { Torr }
$$

which is a factor of 30 to 300 below the pressure in the chamber. High speed shutters (for example spinning disks with holes) could open a $10 \mathrm{~cm}$ diameter hole for a period as short as $2 \mathrm{~ms}$, so the beam line would only be open for $1 \%$ of the time if the system rep rate was $5 \mathrm{~Hz}$. It is only open for the low pressure residual gas, i.e., the high pressures following the explosion are blocked.

The gas volume which is passed by the open shutiers is characterized by molecular flow (long mean free path) and can be readily removed by pumping except for the line of sight fraction. This fraction can be reduced to a few percent of the passed volume if the transition zone is long enough; we assume for the study that a length equal to ten times the beam radius is adequate. Hence the transition length formula: $l_{t}=10 \theta \cdot$ [Reactor Radius $\left.\left(R_{c}+X\right)\right]$. The cost of this section is set at $500 \mathrm{k} \$$ per line.

The development of a transition zone design is a cricical item for HIF.

\section{Beam Transport within The Reactor}

\subsection{Reactor Environment}

The system model defines a reactor chamber of radius $R_{c}=5-10 \mathrm{~m}$ surrounded by a $\mathrm{Li}$ blanket and shielding of total thickness $\mathrm{X}=2 \mathrm{~m}$. The beamlets imust pass through whatever residual gas remains in this zone as they converge towards the pellet. An additional bean line length of $\sim 1 \mathrm{~m}$ between the final magnet and the shielding is occupied by pumping ports and shutters required to prevent a significant amount of gas from reaching upstream into the final focus lenses. No restrictions based only on gas fill are specifically incorporated into the system model. However, it is assumed that $\mathrm{a}$ gas density no greater than $\sim 3 \times 10^{14} \mathrm{~cm}^{-3} \mathrm{Li}$ (equals $10^{-2}$ Torr $\mathrm{Li}$ or equivalent other vapor at $300^{\circ} \mathrm{K}$ ) is present at the moment of beamlet transit. Since pressures in the range 1 -10 Torr appear immediately following an explosion this implies the presence of a very powerful self pump-down of the chamber to match the repetition rate of $\sim 1-10 \mathrm{~Hz}$. The difficulties associated with densitios higher than $3 \times 10^{14} \mathrm{~cm}^{-3} \mathrm{Li}$ are: (a) gas flux into final fosus lenses, (b) filamentation instability and possibly the two-stream instaoility, which are treated below, and (c) possible beam spot spreading from stripping. Limitations due to beam scattering and energy loss set in at $n_{g} \geq 3 x$ $10^{16} \mathrm{~cm}^{-3}$ and are not relevant here. Fortunately the reactor types considered here [Granular Wall, Wetted Wall, HYLIFE (Li jets), and Magnetically Protected Dry Wall] all appear to be potentially capable of meeting this requirement. An interesting contrast is provided by the HIBALL chamber (not considered here), which employs a Li-Pb layer. This special surface pumps down the chamber to $\sim 1011 \mathrm{cat}^{-3} \mathrm{~Pb}$ vapor at a $5 \mathrm{~Hz}$ shot rate. A brief discussion of stability and stripping follows.

\subsection{Stripping}

The cross section for gas stripping of the beam ions is approximately given by 


$$
\begin{gathered}
\sigma_{s} \approx \frac{2.45 \times 10^{-18} \mathrm{~cm}^{2}}{B^{2}}\left(\frac{Z_{i}}{92}\right) \exp \left(-.0632^{\star}\right), \\
z_{i}=\text { atomic no. of ion } \\
z^{*}=\text { stripped state of ion. }
\end{gathered}
$$

Here we have used the numerical $f$ it by Stroud( 7$)$ for $\mathrm{u}^{238}$ on Li, generalized to apply to other heavy ions by incorporating the factor $\left(z_{i} / 92\right)$. For low $Z^{\star}$, a typical value is ( $10 \mathrm{GeV}, \mathrm{U}^{238}$ ).

$$
\sigma_{\mathrm{s}} \approx 2.7 \times 10^{-17} \mathrm{~cm}
$$

A stripping length is defined:

$$
\ell_{S}=\frac{1}{n_{g} \sigma_{s}}=(370 \mathrm{~cm}) \frac{10^{74}}{n_{g}}
$$

An average stripped state of approximately

$$
\overline{Z^{*}}=\mathrm{Z}_{\text {initial }}^{\star}+\frac{\mathrm{R}_{c}+\mathrm{X}}{\ell_{s}}
$$

results as the ions approach the pellet. If $\mathrm{n}_{\mathrm{g}}$ is taken as $3 \times 10^{14}$ $\mathrm{cm}^{-3}$ then, since $R_{c}+X$ is expected to be on the order of $10 \mathrm{~m}$, it is clear that as many as ten electrons are removed in addition to the initial state q.

The consequences of stripping in the chamber are unclear at present. The beam current increases as $Z^{\star}$, and the rigidity decreases as $1 / Z^{\star}$. Hence we expect the stripped ion beam to be more easily disrupted by beam-plasma instabilities. These are discussed below. A second concern is that the beam will not focus to the desired small spot radius due to increased space charge forces. The few estimates made to date of this effect suggest that the problem is reduced or eliminated by the fact that electrons stripped from the ions travel with the beam and neutralize the increased space charge and current. The dangerous possibility is that, since there will be a spread in charge states, the ions will be deflected by varying amounts in the residual self electric field of the beam and the spot size will be spread. Research on this topic--dynamics of the beam envelope in the gas 
environment including the statistical effects of stripping and neutralization--has been inadequate and has been identified by HIFSA as one of the mosic important areas for future simulation and experiment. If stripping is found to be unacceptable in the considered reactor designs then either some other propagation mode which is insensitive to stripping must be considered or a reactor chamber of the HIBALL type $\left(n_{\mathrm{g}} \ll 10^{14}\right)$ must be considered.

\subsection{Eilamentation Instability}

The filamentation mode is a serious concern for high pressure reactors ( $P \geq 10^{-2}$ Torr $\left.L i\right)$. If the beam ions strip to a sufficiently high average charge state and the beam is also neutralized by background electrons, then magnetic pinches can grow during propagation to the pellet and disrupt the convergence processes. A previous analysis of this phenomena(14) gave the safety condition

$$
\alpha=\frac{\omega_{b}{ }^{R} c}{c} \leq 3 \text {, }
$$

where $\omega_{b}$ is the plasma frequency of the beamlet evaluated at the chamber wall, $c$ is the speed of light, and $R_{c}$ is the chamber radius. Because of convergence effects the total mode growth is only on the order of exp $(\alpha)$ $<20$. The mean stripped charge state $Z^{\star}$ is used to evaluate $\omega_{b}$.

$$
\omega_{b}=\left(\frac{n_{b} z^{2} e^{2}}{\varepsilon_{0} m_{0} A}\right)^{1 / 2}
$$

where $n_{b}$ is the beamlet's number density. Using convenient system parameters we have the safety condition

$$
W_{\text {beamlet }} \leq(33 M J)\left(\frac{A}{Z^{*}}\right)^{2} B^{3} \tau_{n s} \theta^{2} .
$$

There is little problem provided $A / Z^{\star} \geq 20$. We estimate for stripping by Li vapor

$$
\underline{Z^{*}} \geq \frac{2.5 m}{R_{c} P_{\text {Torr }}}
$$

so no problem is expected below $\sim 10^{-2}$ Torr, which is the case for the present study. If higher pressures are contemplated then this subject should be given renewed attention. 


\subsection{Two Stream Mode}

Prior to 1985 it was generally believed that unstable two-stream modes eliminated the possibility of heavy ion beams propagating in a background pressure of $10^{-4}-10^{-1}$ Torr $\mathrm{L}$. The analysis of converging beams by P. Stroud $(7)$ has reversed this opinion and for HIFSA we now (optimistically) assume that there is no restriction on pressure from this consideration.

The standard analysis for non-converging beams uses a Fourier decomposition in longitudinal variable $(z)$ and time $(t)$ :

$$
\begin{aligned}
& \text { Perturbed } \\
& \text { quantities }
\end{aligned}
$$

where $k$ is the wave vector and $\omega$ is the frequency. The resulting dispersion relation for the plasma electron-beam ion mode is

$$
1=\frac{\omega_{b}^{2}}{\omega^{2}}+\frac{\omega_{p}^{2}}{(\omega-k v)^{2}}
$$

where $\omega_{b}$ and $\omega_{p}$ are respectively the beam and electron plasma frequencies, and $v$ is the beam velocity. Rapid growth orcurs for $\omega \approx \omega_{p}$ and

$$
k \approx \omega / v
$$

The maximum growth rate in this case is $(.6873)\left(\omega_{p} \omega_{b}^{2}\right)^{1 / 3}$, and only non-linear effects can result in saturation. When convergence of the beam envelope is taken into account this simple (and disastrous) picture is changed because the resonart condition does not persist with distance. The plasma frequencies $\left(\omega_{b}, \omega_{p}\right)$ both increase as the beam converges and any particular unstable wave number $k$ is quickly swept through resonance. The reader is referred to the article by stroud for details; the relevant conclusion is that at typical HIF parameters, less than $1 \%$ of beam ions are deflected from the desired spot at pressures at least up to $3 \times 10^{-3}$ Torr Li.

\section{Sequence of Calculation}

The HIFSA system code requires as input a set of parameters such as ion mass number and net electric power, and also the specification of major features such as pellet and reactor type. A conceptual power plant design is then computed, including cost breakdowns, cost of electricity, size of components, and dependent physical parameters such as spot radius. The 
entire ensemble of designs computed from a broad range of input parameter sets is stored in a data base, which may be searched for a minimum COE subject to specified constraints. Final focus constraints play a large role in the system definition. In this section we give a brief outline of the way these constraints typically enter into the computational sequence (there are several alternatives.)

A net electric output of $1000 \mathrm{~kW}$ (for example) is desired, so a fusion power $P_{f}=4000 \mathrm{MW}$ is tentatively selected. A repetition rate ( $r$ ) is also specified, which fixes the fusion yield $Y=P_{f} / r$. A target type has also been specified; we assume here that the Lindl-Mark(15) single shell gain curves are applicable. These curves relate gain (Q) to the net beam energy on target and the quantity $\gamma_{p}=r_{S}^{3 / 2} R$ where $R$ is ion range in the pellet. The value of $\gamma_{P}$ is specified, and $W=Y / Q$ is inferred from the gain curves. The required net beam power $\left(P_{b}\right)$ is also determined from the Lindl-Mark formulation.

Some major beam parameters are now determined. Mass number (A) and charge state ( $q$ ) have been specified at input but ion energy $\left(T_{0}\right)$ must be selected from a band of permissible values such that the Lindel-Mark spot size condition

$$
\text { (.1) } r_{s}<w_{M J}^{1 / 3}<(.2) r_{s}
$$

is satisfied. the range-energy relation then determines $R$, and spot radius is given by

$$
r_{S}=\left(\gamma_{p} / R\right)^{2 / 3}
$$

The relativistic parameter $B Y$ is determined from $T_{0}$ and $A$ :

$$
B Y=\left[2\left(\frac{T_{0}}{A M_{0} C^{2}}\right)+\left(\frac{T_{0}}{A m_{0} C^{2}}\right)^{2}\right]^{1 / 2},
$$

and magnetic rigidity in final focus is

$$
[B p]=B Y A m_{0} c / q e .
$$

Net beam current (electrical) is

$$
I_{\text {net }}=\frac{P_{b} q e}{T_{0}} \text {, }
$$

however the current per beamlet $I=I_{\text {net }} / H_{0}$ is not determined until the number of beamlets $N_{0}$ is fixed. 
The beamlet cone half angle ( $\theta$ ) must be determined at this point. First a limit $\left(\theta_{\max }\right)$ is fixed by the aberration condition

$$
\theta_{\max }=(.15)\left(\frac{r_{s}}{\rho}\right)^{1 / 4} .
$$

where $\rho$ is the radius of curvature in the final quadrupole in the final focus beamline. We ușe

$$
\rho=\frac{\left[B_{p}\right]}{B_{p}}
$$

with specified $B_{p}$ (typically $B_{p}=1.0 T$ ). The actual value of $\theta$ is the smaller of $\theta_{\text {max }}$ and the optimum value

$$
\theta=\frac{r_{s}}{\sqrt{2} 8 L \Delta p / p}
$$

The momentum spread is specified (typically $\Delta p / p= \pm 10^{-3}$ ) and the distance to the center of the $f$ inal lens $(L)$ is determined from the sum of the specified reactor cavity and blanket radii $\left(R_{c}+X\right)$, and half the final lens length $(\ell)$ :

$$
L=(1+10 \theta)\left(R_{c}+X\right)+\ell / 2,
$$

with the factor $10 \theta \approx .1$ inserted to allow room for shutters and pumping.

The final lens aperture $R_{f}$ and magnet length are determined simultaneously with L using the thin lens formulas

$$
\begin{gathered}
\ell=\frac{R_{f}[B \rho]}{L B}, \\
R_{f}=(1.1) \theta(L+\ell) .
\end{gathered}
$$

At this point the final emittance $(\varepsilon)$ is determined from $\theta$ and $r_{s}$ :

$$
\varepsilon=r_{s} \theta / \sqrt{2},
$$

where the factor of $\sqrt{2}$ reflects the fact that momentum spread and emittance are assumed to play an equal role in determining $\mathbf{r}_{\mathbf{s}}$. Normalized emittance is $\varepsilon_{n}=B_{Y} \varepsilon$. This is the value used in the calculation of 
accelerator parameters. In case this value of $\varepsilon_{n}$ exceeds the tabulated values used in the LIACEP survey of the linac parameters we use its maximum tabulated value.

The beamlet current (I) and hence beamlet number $\left(\mathrm{N}_{0}\right)$ is now determined from the perveance condition

$$
N_{0} \geq(.138)\left(\frac{q}{A}\right)^{2} \frac{W M J}{\theta^{2}(B Y)^{5} \tau_{n S}}
$$

If this inequality allows a smaller number of beamlets than is assumed for the Linac then the Linac number $(N)$ is used, i.e. there may be splitting, but no merging is considered after acceleration.

Having determined $N_{O}, L, \Theta$ and $[B p]$, the lengths, apertures and field strengths of the final focus elements are determined (total train length is $8 \mathrm{~L}$ ). These quantities are used to obtain a cost estimate which is entered into the system cost table. The lengths and costs of the transport lines are estimated in similar fashion. The remainder of the calculation determines the cost of the accelerator, balance of plant, reactor, etc. as well as the resultant net electrical output. If this output differs from the original input, the calculation is repeated with an increased or decreased value of fusion power. 


\section{$\underline{\text { References }}$}

1. R. L. Martin, "Emittance Limitations in Heavy Ion Fusion," Nucl. Instruments and Methods, 187, p. 271 (1981).

2. B. Badger, et al., "HIBALL-II, An Improved Conceptual Heavy Ion Beam Driven Fusion Reactor Study," Kernforschungszentrum Karlsruhe Report KFK 3840, Karlsruhe, FRG (July, 1985).

3. Proceedings of the Heavy Ion Fusion Workshop Held at Argonne National Laboratory - 1978, (R. C. Amnold, editor), ANL-79-41, p. 148.

4. J. A. Blink, W. J. Hogan, J. Hovingh, W. R. Meir, and J. H. Pitts," The High-Yield Lithium-Injected Fusion-Energy (HYLIFE) Reactor," LLNL report UCRL-53559 (1985).

5. J. H. Pitts and I. Maya, "The Cascade Inertial-Confinement-Fusion Power Plant," Proc. 11th Symp. on Fusion Engineering, p. 130, Austin, TX (Nov. 1985).

6. J. H. Pendergrass, T. G. Frank, I.O. Bohachevsky, "Modified Wetted WaIl Inertial Fusion Reactor Concept," Proc. 4th Topical Meeting, Technology of Controlled Nuclear Fusion, King of Prussia, Pa, Oct. 1980, CoNF 801011 , p. 1131, USADOE, (July 1981).

7. P. Stroud, "Streaming Hodes in Final Beam Transport for Heary Ion Fusion," Laser and Particle Beans, 4, Part 2, Pg. 261, 1986.

8. I. Brown, "An Intense Metal Ion Beam Source for Heavy Ion Fusion," Proceedings of the International Symposium on Heavy Ion Fusion, AIP Conf. Proc. 152, p.207.

9. J. W-K Mark et al., "Studies on Longitudinal Beam Compression In Induction Accelerator Drivers," Proceedings of the International Symposium on Heavy Ion fusion, AIP ccnf. Proc. 132, p. 227.

10. C. L. Olson, "HIF Transport Issues for $\mathrm{P}>10^{-3}$ Torr and $\mathrm{Z}>1$, "Proceedings of the International Symposium on Heavy Ion fusion, AIP Conf. Proc. 152, p. 215 .

11. E. D. Courant, "Power Transport in Quadrupole or Solenoid-Fucussing Systems," Proc. ERDA Summer study of Heavy Ions for Inertial Fusion, p. 72, LBL-5543, Berkeley, CA (Dec., 1976).

12. E. P: Lee, "Transport of Intense Ion Beams," Proc. Second International Conference on Charged Particle Optics, Albuquerque, NM, June 1986. To be published in Nuclear Instruments and Methods in Physics Research.

13. D. Neuffer, "Geometric Aberrations In Final Focussing For Heavy Ion Fusion," Proceedings of the Heavy Ion Fusion Workshop Held at Argonne National Labcratory - 1978, (R. C. Arnold, editor), ANL-79-41, p. 333. 
14. E. P. Lee, et al., "Filamentation of a Heavy-Ion Beam in a Reactor Vesse1," Phys. Fluids 23 (10), p. 2095, 1980.

15. J. D. Lindl et al., Review of Target Studies for Heavy Ion fusion," Proceedings of the International symposium on Heavy Ion fusion, AIP Conf. Proc. 152, p. 89. 